outcome of interest. Predictor variables studied were age, sex, duration prior to ART initiation and duration on ART, ART regimen, orphan status, baseline WHO staging and adherence. Bivariate analysis and multivariate logistic regression were used to establish determinants of non-suppression.

Results We included 1,066 CLHIV of whom 51.3\% were female, median age was 7.5 years (IQR 5.7-9) and a quarter were orphans. Median duration on ART was 51 months (IQR 31-79), 20.4\% were on second line ART regimen with an overall viral suppression rate of $88 \%$. Children who had been on ART for a longer duration ( $>5$ years) were more likely to be suppressed $[\mathrm{aOR}=0.38,(95 \%(\mathrm{CI})=0.17-0.86), \mathrm{p}=0.02]$. A protease inhibitor containing regimen was associated with nonsuppression on bivariate analysis $[\mathrm{OR}=2.43$, $(95 \% \mathrm{CI}=1.04-$ $5.65), \mathrm{p}=0.039]$ however this was not significant in multivariate analysis. Non-adherence to ART increased five-folds the odds of non-suppression $[\mathrm{aOR}=5.47$, (95\% CI $=1.12-26.69)$, $\mathrm{p}=0.035]$ whereas those who were orphans were more likely to be suppressed $[\mathrm{aOR}=0.56, \quad(95 \%$ CI $=0.37-0.86)$, $\mathrm{p}=0.007]$.

Conclusion CLHIV within our study population had sub-optimal viral suppression. Innovative strategies to address adherence remains crucial in addressing non-suppression.

Disclosure No significant relationships.

\section{P167 PCR DETECTION OF HIV PROVIRAL DNA IN BRAIN TISSUES FROM DEAD HIVIAIDS IN ZAMBIA}

${ }^{1}$ Lishi Bai*, 'Chun Huang, ${ }^{1}$ Ruolei Xin, ${ }^{2}$ Wood Charles. 'Beijing Center for Disease Prevention and Control, Beijing, China; ${ }^{2}$ University of Nebraska-Lincoln, Nebraska Center of Virology, Lincoln, USA

\subsection{6/sextrans-2019-sti.326}

Background The prevalence of HIV-associated neurocognitive disorders (HAND) was highly prevalent In Zambia. But very little is known about the effect of ART on HIV subtype C associated neurological disease.

Methods Brain and lymphnotes tissues: from autopsies of dead HIV+/AIDS patients, provided by local hospitals. First, frozen tissue blocks were cracked, DNA was extracted. Then, Env V2-V5 region of HIV proviral DNA was amplified by PCR with clade $\mathrm{C}$ specific primers. 2.0 $\mu \mathrm{g}$ of template DNA was employed with Fast Start HF and platinum pfx DNA. Finally, phylogenetic trees were drawn.

Results Out of 12 HIV/AIDS cases detected, 6 cases were found to be HIV DNA positive for at least 1 positive in total 7 different brain tissues (FL,PL,TL,OL,H,C,BG), and their lymphnote samples were also HIV positive the same time. So HIV infection rate of brain was $50 \%$. For the 2 ART non-treated cases, $85.7 \%(12 / 14)$ of the samples of different tissue was HIV positive, while it was $17.6 \%(6 / 34)$ in 7 ART treated cases. All DNA sequences were blasted as HIV subtype $\mathrm{C}$, and clustered with subtype $\mathrm{C}$ reference strains.

Conclusion In this study, the HIV infection rate in all brain tissue samples in untreated cases $(87.5 \%)$ was much less than that in treated cases(17.6\%), It suggested that ART may help control the spread of HIV infection in brain tissues.

Disclosure No significant relationships.
P168 MULTIDRUG RESISTANT TUBERCULOSIS IN TB/HIV COINFECTED PATIENTS IN RIVERS STATE, NIGERIA

${ }^{1}$ Mary Alex-Wele*, ${ }^{2}$ Nneka Onyejepu, ${ }^{1}$ Orikomaba Obunge. ${ }^{1}$ University of Port Harcourt teaching Hospital, Medical Microbiology and Parasitology, Port Harcourt, Nigeria; ${ }^{2}$ Nigeria Institute of Medical Research, Centre for Tuberculosis Research, Lagos, Nigeria

10.1136/sextrans-2019-sti.327

Background Tuberculosis poses a serious health problem worldwide with a high mortality rate, especially in immunocompromised individuals. The emergence of drug-resistant forms of tuberculosis further burdened with high prevalence of HIV threatens to make this important human disease difficult to manage. Nigeria is currently listed among the 30 high burden countries for TB, TB/HIV and DR-TB. This study was carried out to determine Mycobacterium tuberculosis resistance pattern to first-line anti-TB drugs (rifampicin and isoniazid) in TB patients who were HIV seropositive in Rivers State.

Methods Two hundred and sixty HIV sero-positive patients $\geq$ 18 years old were recruited from three health care facilities in Rivers State. The subjects were separated into two groups consisting of $130 \mathrm{~TB} / \mathrm{HIV}$ co-infected patients on anti-tuberculosis treatment and $130 \mathrm{HIV}$ seropositive patients, suspected of having tuberculosis and yet to commence anti TB treatment. Sputum samples were processed by line probe assay (MTBDRplus by HAIN Lifescience). Analysis were carried out with the SPSS v20 software.

Results Of the 260 recruited HIV seropositive subjects, 159 were positive for TB: 127 from the treatment exposed group and 32 from the treatment naïve group. Among all the TB/ HIV co-infected subjects, MDR-TB was detected in $10.1 \%$ $(16 / 159)$ of the study subjects. Among the treatment experienced group, MDR-TB was detected in $11.0 \%$ (14/127), INHmonoresistance in $15.7 \%(20 / 127)$ and RIF-monoresistance in $11.8 \%(15 / 127)$ while $6.3 \%(2 / 32)$ of treatment naïve subjects had MDR-TB, 12.5\% (4/32) had INH-monoresistant TB and 6.3\% (2/32) had RIF-monoresistant TB.

Conclusion Primary MDR-TB was prevalent in Rivers State $(6.3 \%)$. This implies a high level of ongoing transmission of MDR-TB in TB/HIV co-infected individuals within the community. The DOTS program needs to be strengthened to capture TB/HIV co-infected individuals early enough to manage them promptly.

Disclosure No significant relationships.

\section{P169 ASSOCIATION BETWEEN CXCR4 AND TRAIL PATHWAY EXPRESSION IN CD4 T LYMPHOCYTES FROM HIV+ ART- NAÏVE PATIENTS}

${ }^{1}$ Sarah Ratkovich-Gonzalez*, 'Judith De Arcos Jimenez, ${ }^{2}$ Luz Gonzalez-Hernandez, 'Jaime Andrade-Villanueva, ${ }^{1}$ Monserrat Alvarez-Zavala, ${ }^{1}$ Karina Sanchez-Reyes. ${ }^{1}$ Universidad de Guadalajara, Guadalajara, Mexico; ${ }^{2}$ Hospital Civil Fray Antonio Alcalde, Unidad De Vih, Guadalajara, Mexico

\subsection{6/sextrans-2019-sti.328}

Background HIV infection is characterized by immune cells depletion, apoptosis is one of the main mechanisms described. It has been reported that, the union of HIV to coreceptor CXCR4 can induce Fas independent apoptosis on T cells. In a few in vitro studies it has been proved that stimulation of 